\title{
Managing Complexity in Chronic Care: An overview of the VA State-of-the-Art (SOTA) Conference
}

\author{
Kevin B. Weiss, $M D^{1,2,3}$ \\ 'Center for Management of Complex Chronic Care, Hines VA Hospital, Hines, IL, USA; ${ }^{2}$ Institute for Healthcare Studies, Northwestern University \\ Feinberg School of Medicine, Chicago, IL, USA; ${ }^{3}$ Hines VA Hospital, Hines, IL, USA.
}

INTRODUCTION: The aging of the population and the increasing prevalence of multiple chronic illnesses, along with multiplying options for clinical management, pose great challenges to both the Veterans Health Administration (VHA) and the broader US health care system. Developing effective and efficient health care for persons with complex and multiple medical conditions is a national priority. Therefore, research in this area is critically important. In 2006, the VHA Health Services Research and Development Service held a state-of-theArt (SOTA) conference titled "Managing Complexity in Chronic Care" to clarify our current understanding of the management of complex chronic conditions and suggest directions for research to better address this important problem.

OBJECTIVE: This article provides an overview of the major findings from that conference, including major presentations, summaries of the workgroup deliberations, and a list of research topics that were thought to be of highest importance to advancing our ability to provide medical care for persons with complex chronic care needs.

KEY WORDS: chronic illness; co-morbidity; clustered illness; veteran; conference proceeding.

J Gen Intern Med 22(Suppl 3):374-8

DOI: $10.1007 / \mathrm{s} 11606-007-0379-\mathrm{x}$

(c) Society of General Internal Medicine 2007

I $\mathrm{t}$ is estimated that more than 120 million persons in the United States have a chronic health condition, and $24 \%$ of those have 3 or more conditions. ${ }^{1}$ Persons with multiple chronic conditions are at greater risk of disability and activity limitations. Health care spending for a person with 1 chronic condition is, on average, 2 and a half times greater than spending for someone without any chronic condition, whereas spending is almost 15 times greater for someone with 5 or more chronic conditions. Currently, nearly all of Medicare spending $(96 \%)$ is on behalf of people with multiple chronic conditions. Per capita Medicare expenditures were found to increase from \$211 annually for persons without chronic conditions to $\$ 13,973$ for persons with 4 or more types of chronic conditions. $^{2}$

The challenge to the Department of Veterans Affairs (VA) and other parts of the US health care system is how to best serve the needs of patients with these complex chronic care needs.
There are a number of basic concerns related to patients with multiple chronic illnesses and other complex chronic care needs. First is whether the care needs of persons with multiple chronic illnesses are more or less likely to be met when the chronic illness issues are unrelated. In 1 of the larger studies of this question, Redelmeier, et al. ${ }^{3}$ examined drug therapy for an unrelated disorder among more than 30,000 patients with diabetes, 56,000 with emphysema, and 17,000 with psychotic syndromes in the province of Ontario, Canada. In each case, persons with the condition were less likely to receive drug therapy for the unrelated disorder than persons without the conditions, suggesting that the presence of 1 chronic condition decreased the likelihood that another chronic condition would be treated.

An area of particularly active research in the care of persons with multiple chronic illnesses has been among physical and mental disorders. Perhaps the most well-studied area within these 2 major groups of chronic illnesses has been diabetes and depression. A metaanalysis examining these comorbidities suggests that there is a strong inverse relationship between good glycemic control and active depression. ${ }^{4}$ Moreover, a recent analysis of the US Medical Expenditure Panel Survey suggested that adequacy of mental health treatment varied by the specific type of physical co-morbidity and condition of interest. ${ }^{5}$ Studies such as these are still infrequent, yet they suggest areas of further study and potential clinical care management that may lead to promising treatment strategies.

VA is particularly concerned with chronic care as a result of the increasing numbers of aging veterans who are coping with multiple chronic diseases. And although VA offers a comprehensive set of chronic care services and has a strong tradition in primary care, a patient with multiple chronic conditions or other complex care needs will have to negotiate a multitude of health care providers, ancillary services, and administrative tasks to get optimal care. In addition, for many of VA's older patients, chronic care needs are further complicated by the dual use of both VA services and private sector health care available through Medicare benefits. Many of VA's patients have dual use of VHA and Medicare-sponsored private health care services. Devising the best possible patient-centered care for patients with complex chronic care needs requires substantive mechanisms for continual patient and caregiver input and feedback.

In addition to its role as a comprehensive provider of services for the general veteran population, VA also has a unique role in managing complex illness for active military service members. More than 1 million US military service members have served or are serving in Operation Iraqi Freedom or Operation Enduring Freedom. These service 
members may be eligible to receive health care from the VA while serving on active duty or upon separating from active duty. Since 1982, Congress has authorized VA to provide health care services to service members in time of war or national emergency, when the Department of Defense (DoD) may have insufficient resources to care for casualties. As of December 2005, VA has been serving active duty service members with serious injuries, such as traumatic brain injuries and other complex trauma, by providing medical and rehabilitative care at VA polytrauma rehabilitation centers. ${ }^{6}$

Thus, VA's Office of Research and Development convened a state-of-the-art (SOTA) conference to advance knowledge about managing complexity in chronic care and to make recommendations that address both system and patient improvements within VA and the greater health care community. This SOTA which brought together experts on chronic care from both within and outside the VA, focused on what is needed in clinical care, research, and education to help create a health care system that will best manage the complexity associated with veterans with multiple chronic illnesses.

To provide a background for conference participants before they convened in their respective workgroups, a number of papers were commissioned to examine issues that range from characterizing the burden of multiple chronic conditions to the business case and information systems needed to optimize care for complex patients. These papers were revised after the SOTA conference to reflect the workgroups' findings and research recommendations, and are included in this Supplement.

The SOTA conference was organized around workgroups dedicated to the following topics:

- Identifying the Patient with Complex Chronic Illness,

- Self-Management for Patients with Complex Chronic Illnesses,

- Developing the Evidence and Knowledge Base for Managing Patients with Complex Chronic Illness,

- Improving Systems to Manage Complex Chronic Care

- Informatics for Complex Chronic Care, and

- Linking Patient and System Strategies for Managing Complexity.

The following are summaries of the findings and research recommendations from these workgroups.

\section{IDENTIFYING THE PATIENT WITH COMPLEX CHRONIC ILLNESS}

Improving the management of patients with complex chronic illnesses requires the establishment of explicit definitions and criteria for identifying these patients, as well as feasible methods for operationalizing these criteria. This workgroup was charged with identifying research needs and recommendations for policy and practice initiatives to advance the development and implementation of patient screening and identification tools.

First, the Workgroup discussed the need to identify a patient as complex because this type of patient is one for whom clinical decision making and required care processes are not routine or standard. For complex patients, many recommendations from evidence-based medicine are unlikely to apply in a straightforward manner because of "exceptions" such as: multiple interacting chronic conditions, other co-morbid conditions that complicate the management of the focal chronic disease, and socioeconomic factors such as homelessness or the absence of adequate family caregivers or other support systems. The Workgroup discussed the need for an expanded list of core elements of complexity (i.e., medical/biological/ genetic, socioeconomic, environmental, cultural, behavioral). They also developed a preliminary list of required services, based on the needs of these patients and the ways in which standard services (offered to patients who are not identified as complex) would be inappropriate or insufficient for different groups of complex patients.

Based on their discussions, this workgroup proposes that the research agenda focus on advancing the identification and characterization of high-risk cohorts of patients, or patient clusters, with multiple complex chronic illnesses, who also may be affected by social complexities (i.e., homelessness). They recommend developing a priority list of these patient clusters for targeted interventions.

\section{SELF-MANAGEMENT FOR PATIENTS WITH COMPLEX CHRONIC ILLNESS}

Complex patients with multiple chronic conditions require unique self-management support. Although successful selfmanagement support can be effectively delivered in "stand alone" programs for persons with a single disease, recent evidence suggests that long-term benefits may require an ongoing collaborative process between patients and professionals, ${ }^{7,8}$ as well as incorporating families. ${ }^{9}$ This workgroup focused on defining self-management for patients with complex chronic illnesses, identifying who is responsible for their care (e.g., patients vs health care system), and who should deliver that care (e.g., physician vs non-physician). The group also discussed potential barriers to self-management.

Whereas increasing attention has been devoted to selfmanagement, substantial barriers impede the implementation and dissemination of these programs. Barriers to selfmanagement occur at multiple levels: patients (and their environment), providers, and the system. Patient-level challenges include prioritizing multiple demands, acquiring individualized care plans, and using new technologies to optimize self-management. Provider-level barriers to facilitating selfmanagement may include inadequate staff, time, and resources, as well as a lack of appropriate treatment guidelines for complex chronic illnesses. Barriers linked to systems may include a lack of reimbursement from third-party payers for specific selfmanagement support tasks. Therefore, implementation of complex chronic disease self-management is likely to require multiple implementation modalities that can address these barriers.

Based on their discussions, this workgroup proposes that future researchers consider experimentation with new and existing technologies and models of care delivery (i.e., group visits, peer support, telemedicine) that may provide opportunities for improved self-management. Moreover, the development of toolkits for both providers and patients that cover both process and content would be helpful in further disseminating successful self-management methods and material.

\section{DEVELOPING THE EVIDENCE BASE FOR MANAGING COMPLEX CHRONIC ILLNESS}

There is a substantial evidence base for managing individual diseases, but we lack evidence about using this knowledge to 
care for the complex patient. This workgroup identified key issues that must be investigated to develop an evidence base for managing multiple chronic illnesses.

These key issues include: 1) how to engage a variety of stakeholders (i.e., patients, clinicians, managers, behavioral scientists, and systems engineers); 2) developing improved research methods to examine the issues surrounding complex patients (i.e., better patient-centered outcome measures, clinical trials that focus on complex patients); 3) developing evidence-based guidelines that address patients with multiple chronic conditions; 4) evaluating the effectiveness of current models of care for complex patients, and further exploration of newer models; 5) developing methods to educate and train health care professionals, including multidisciplinary teams; and 6) determining how to best engage patients and their caregivers in chronic illness management.

Multidisciplinary research teams are needed to address these issues, in addition to supporting efficacy and effectiveness studies that will increase the evidence base to support guidelines that are adaptive to chronic complex illnesses. This group also recommended that research be prioritized based on its potential to translate findings into practice and inform policy decisions.

\section{IMPROVING SYSTEMS TO MANAGE COMPLEX CHRONIC CARE}

A system consists of the interaction, interdependencies, and behavior of multiple entities and persons that share a goal of producing a desired output. These entities interact with one another in different manners-cooperative, collaborative, competitive, or contradictory. This Workgroup identified 3 steps that are necessary to improve system performance.

First, desired goals or outputs of the system must be identified-in this case, to enhance value provided to patients. Value is defined as quality divided by cost and can be enhanced by improving quality and reducing costs. In the management of complex chronic care patients, high quality is achieved through maximizing functional status, quality of life, and patient satisfaction. Costs constitute not only dollars expended in care provision, but also risk exposure to adverse events. This exposure can be minimized by reducing adverse events associated with care, eliminating unnecessary care, and enhancing patient safety. The second step is to identify characteristics of high-performance systems. Critical characteristics include having a patient-centric orientation, wherein autonomy and independence are fostered and decisional control over treatment rests with patients who have access to aids that enhance values-consistent decision making. And the final step is to adopt high-performance characteristics. Because of the interdependencies inherent in systems, it is important to understand the implications and potential unintended consequences of adopting particular characteristics, a goal that can be achieved through research.

Based on their discussions, this workgroup recommends a research agenda that defines high performance care for patients with chronic complex illnesses and how that care will be measured. In addition, researchers must determine the best way to implement new models of care.

\section{INFORMATICS FOR COMPLEX CHRONIC CARE}

There is great potential in clinical informatics and its ability to help facilitate complex chronic care. Currently, efforts are underway to consider the best uses of information technology to support the unique aspects of complex chronic care within the VA health care system. This workgroup identified key areas that need to be addressed by researchers to best utilize information technology for this purpose.

These key areas include: 1) determine how information systems can improve care for complex chronic illness during and between patient visits; 2) design clinical information systems to promote communication and information sharing among clinicians treating patients with complex chronic illness; 3) identify how to use informatics to improve patient self-management; 4) ascertain how to use informatics to encourage patients with complex chronic illness to share information with their providers between visits; 5) determine the type of aggregate information that should be captured by clinical information systems to help improve quality of care at the provider and system levels; and 6) identify what evidencebased practices in informatics already exist that systems should begin to implement.

This workgroup also made recommendations regarding specific research questions that need to be addressed, such as-Can informatics resources be used to increase patients' participation in their own care? If you develop an informatics system to support communication between providers, does it improve care? And, can informatics increase agreement between patients and providers about goals?

\section{LINKING PATIENT AND SYSTEM STRATEGIES FOR MANAGING COMPLEXITY}

As VA tackles the daunting task of putting systems in place to optimally care for the complex patient, it must maintain a patient-centered approach by constantly asking whether the processes and structures of care make sense from the perspective of the patient and his or her caregivers. This workgroup focused on system-level requirements that would be required to provide optimal, patient-centered care for veterans with complex chronic illnesses.

The key components of the care system are continuity of care across time, care coordination across settings, patient and family engagement, and patient-centered assessment and care planning. This kind of care is best implemented through collaborative team-based approaches. One of the key components of this model of care is a "point person," a specific individual whom the patient and family can easily access and who will get them the answers they need. This person, who can be any member of the team, is also responsible for assuring that the care plan is being implemented and that care across different venues is coordinated. Appropriate guidelines and performance measures targeting the right outcomes also are essential to improving care. For example, it is important to identify common co-occurring conditions (i.e., diabetes and hypertension), compare guidelines for those conditions, resolve any contradictions, and develop cross-cutting guidelines, if needed.

For this type of collaborative, patient-centered care to be successfully implemented and sustained, further research needs to be done. This workgroup recommends that researchers focus on how to measure patient-centered care, as well as how to test cross-cutting guidelines for multiple chronic conditions. 
1. Characterize high risk cohorts of patients with Multiple Chronic Conditions (MCCs) and social complexity, including health services impact. From this work develop a priority list of MCCs and social complexity for targeted interventions.

2. Synthesize/systematically review literature of interventions that relate to MCCs and complex care needs for patients with social complexity.

3. Advance work in outcomes assessment, including measures of comprehensive care needs and optimized outcomes for patients with MCCs.

4. Increase the evidence-base of efficacy and effectiveness studies to support guidelines that are adaptive to MCC and social complexity for high priority complex patients.

5. Development of more optimal performance measures that reflect complex morbidity, including focus on patient self-management and coordination of care.

6. Evaluate systems changes that organize care around MCC and social complexity of illness management such as:

- new team-based strategies for care in complex chronic care management

- new non-MD team member roles increased role in care

o the role of and different designs of an "advance medical home" in managing patients with complex care needs

- the role of care sharing between physician specialties and service lines in optimal management of care

- self-management support, including group-based learning structures

- high performance systems of care for patients with high priority MCCs

- technology assistance for patients with visual, hearing, and other physicial limitations in optimizing complex care management

7. Examine best practices in patient-physician communication strategies for care management decisions for patients with MCCs or with social complexity:

- What are best methods for eliciting patient preferences in light of care complexity, and engaging patient social support structures (e.g. family)?

8. Evaluate new Health Information Technology strategies to support complex care management to advance knowledge of:

- What decision support tools are needed for patients with complex care needs?

- How can patient registries best support care management for patients with MCCs?

- What type of Patient directed HIT tools can be developed for optimizing selfmanagement for such patients?

9. Identify best practices for integration of rehabilitation services into patient management strategies for patients with complex chronic care needs.

Basic and Clinical Science research focus: Enhance understanding of some of the gene environment interactions that lead to some of the more common MCCs.

Figure 1. Research topics in the management of patient with complex chronic care needs identified at the SOTA conference

\section{RESEARCH TOPICS OF INTEREST AND CONCLUSION}

Figure 1 presents a list of recommendations for future research in this area summarized from the efforts of the conference workgroups and general discussion. This list reflects the recommendations of the conference participants. It is not an official VA statement of research priorities in this area. This list is presented to hopefully be useful to the VA, other federal departments and agencies, and private foundations in helping them formulate their research priorities in this area.

The closing discussions for this conference reflected the participants strong belief that research in this area is still in its early stages and that there are substantive opportunities to better understand care, improve outcomes, and enhance value in the care of patients with complex care needs. I believe that the proposed list of research topics can serve as a starting place for new efforts to build an evidence base that supports even better clinical care for these patients.

Acknowledgments: This SOTA was sponsored by the VA's Health Services Research and Development Service. It is important to acknowledge contributions of all the conference participants for their contributions through their ideas and commentary. In particular, a special thank you to the SOTA workgroup leaders who both facilitated their group discussion but also summarized the workgroup deliberations in preparation for this overview, including (listed alphabetically) Hanna Bloomfield, MD, MPH, Hayden B. Bosworth, PhD, Polly Hitchcock Noel, PhD, Denise Hynes, PhD, RN, Brian Mittman, PhD, Bonnie Wakefield, $P h D, R N$, William Weeks, MD, MBA. Also it is important to acknowledge the editorial assistance of Geraldine McGlynn M.Ed. and Diane Hanks in the preparation of this overview. The SOTA conference 
would not have been possible without the expertise and guidance of Ms. Geraldine McGlynn and her staff of the VA's HSR\&D Center for Information Dissemination and Education Resources (CIDER). Lastly, these proceedings of the conference would not have been possible without the support of the VA's HSR\&D service, and the combined efforts of Ms. McGlynn, the staff at CIDER, and the great editorial team at JGIM.

Corresponding Author: Kevin B. Weiss, MD; Hines VA Hospital, 5000 South 5th Avenue, Hines, IL 60141-3030, USA (e-mail: Kevin. Weiss@va.gov).

\section{REFERENCES}

1. Anderson G, et. al. Chronic conditions: making the case for ongoing care. A Chartbook. Partnership for Solutions. September 2004.

2. Wolff JL, Starfield B, Anderson G. Prevalence, expenditures, and complications of multiple chronic conditions in the elderly. Arch Intern Med. 2002;162:2269-76.
3. Redelmeier DA, Tan SH, Gooth GL. The treatment of unrelated disorders in patients with chronic medical diseases. New Engl $\mathrm{J}$ Med. 1998;338:1516-20.

4. de Groot M, Anderson R, Freedland KE, Clouse RE, Lustman PJ. Association of depression and diabetes complications: a meta-analysis. Psychosom Med. 2001;63:619-30.

5. Harman JS, Edlund MJ, Fortney JC, Kallas H. The influence of comorbid chronic medical conditions on the adequacy of depression care for older Americans. J Am Geriatr Soc. 2005;53:2178-83.

6. Government Accounting Office report number GAO-06-794R entitled "VA and DOD Health Care: Efforts to provide Seamless Transition of Care for OEF and OIF Service members and Veterans," US Government Printing Office, Washington, DC., July 2006.

7. Guevara JP, Wolf FM, Grum CM, Clark NM. Effects of educational interventions for self management of asthma in children and adolescents: systematic review and meta-analysis. BMJ. 2003;326:1308-9.

8. Clark NM. Management of chronic disease by patients. Annu Rev Public Health. 2003;24:289-313.

9. Bodenheimer T, Lorig $\mathbf{K}$, Holman H, Grumbach $\mathbf{K}$. Patient selfmanagement of chronic disease in primary care. JAMA. 2002;288: 2469-75. 